\title{
Photonic band-gap structures
}

\author{
E. Yablonovitch* \\ Department of Electrical Engineering, University of California, Los Angeles, Los Angeles, California 90024-1594
}

Received June 17, 1992

\begin{abstract}
The analogy between electromagnetic wave propagation in multidimensionally periodic structures and electronwave propagation in real crystals has proven to be a fruitful one. Initial efforts were motivated by the prospect of a photonic band gap, a frequency band in three-dimensional dielectric structures in which electromagnetic waves are forbidden irrespective of the propagation direction in space. Today many new ideas and applications are being pursued in two and three dimensions and in metallic, dielectric, and acoustic structures. We review the early motivations for this research, which were derived from the need for a photonic band gap in quantum optics. This need led to a series of experimental and theoretical searches for the elusive photonic band-gap structures, those three-dimensionally periodic dielectric structures that are to photon waves as semiconductor crystals are to electron waves. We describe how the photonic semiconductor can be doped, producing tiny electromagnetic cavities. Finally, we summarize some of the anticipated implications of photonic band structure for quantum electronics and for other areas of physics and electrical engineering.
\end{abstract}

\section{INTRODUCTION}

In this paper we pursue the rather appealing analogy ${ }^{1,2}$ between the behavior of electromagnetic waves in artificial, three-dimensionally periodic, dielectric structures and the rather more familiar behavior of electron waves in natural crystals.

These artificial two- and three-dimensionally periodic structures we call photonic crystals. The familiar nomenclature of real crystals is carried over to the electromagnetic case. This means that the concepts of reciprocal space, Brillouin zones (BZ's), dispersion relations, Bloch wave functions, Van Hove singularities, etc. must be applied to photon waves. It then makes sense to speak of photonic band structure (PBS) and of a photonic reciprocal space that has a BZ approximately 1000 times smaller than the BZ of electrons. Because of the periodicity, photons can develop an effective mass, but this implication is in no way unusual, since it occurs even in onedimensionally periodic, optically layered structures. We frequently leap back and forth between the conventional meaning of a familiar concept such as conduction band and its new meaning in the context of PBS's.

Under favorable circumstances a photonic band gap can open up, a frequency band in which electromagnetic waves are forbidden irrespective of propagation direction in space. Inside a photonic band gap optical modes, spontaneous emission, and zero-point fluctuations are all absent. Because of its promised utility in controlling the spontaneous emission of light in quantum optics, the pursuit of a photonic band gap has been a major motivation for studying PBS.

\section{MOTIVATION}

Spontaneous emission of light is a major natural phenomenon that is of great practical and commercial importance. For example, in semiconductor lasers spontaneous emission is the major sink for threshold current and must be surmounted in order to initiate lasing. In heterojunction bipolar transistors, which are all-electrical devices, spontaneous emission nevertheless rears its head. In certain regions of the transistor current-voltage characteristic, spontaneous optical recombination of electrons and holes determines the heterojunction-bipolar-transistor current gain. In solar cells, surprisingly, spontaneous emission fundamentally determines the maximum available output voltage. We shall also see that spontaneous emission determines the degree of photon-number-state squeezing, an important new phenomenon ${ }^{3}$ in the quantum optics of semiconductor lasers. Thus the ability to control spontaneous emission of light is expected to have a major effect on technology.

The easiest way to understand the effect of a photonic band gap on spontaneous emission is to take note of Fermi's golden rule. Consider the spontaneous-emission event illustrated in Fig. 1. The downward transition rate $w$ between the filled and the empty atomic levels is given by

$$
w=\frac{2 \pi}{\hbar}|V|^{2} \rho(E),
$$

where $|V|$ is sometimes called the zero-point Rabi matrix element and $\rho(E)$ is the density of final states per unit energy. In spontaneous emission the density of final states is the density of optical modes available to the photon emitted in Fig. 1. If there is no optical mode available, there will be no spontaneous emission.

Before the 1980's spontaneous emission was often regarded as a natural and inescapable phenomenon, one over which no control was possible. In spectroscopy it gave rise to the term natural linewidth. However, in 1946, an overlooked note by Purcell ${ }^{4}$ on nuclear spin-levels already indicated that spontaneous emission could be controlled. In the early 1970's interest in this phenomenon was reawakened by the surface-adsorbed dye molecule fluorescence studies of Drexhage. ${ }^{5}$ Indeed, during the mid-1970's Bykov ${ }^{6}$ proposed that one-dimensional periodicity inside a coaxial line could influence spontaneous emission. The modern era of inhibited spontaneous 


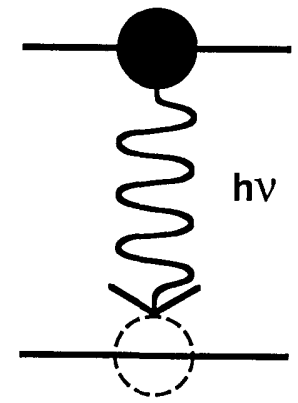

Fig. 1. Spontaneous-emission event from a filled upper level to an empty lower level. The density of final states is the available mode density for photons.

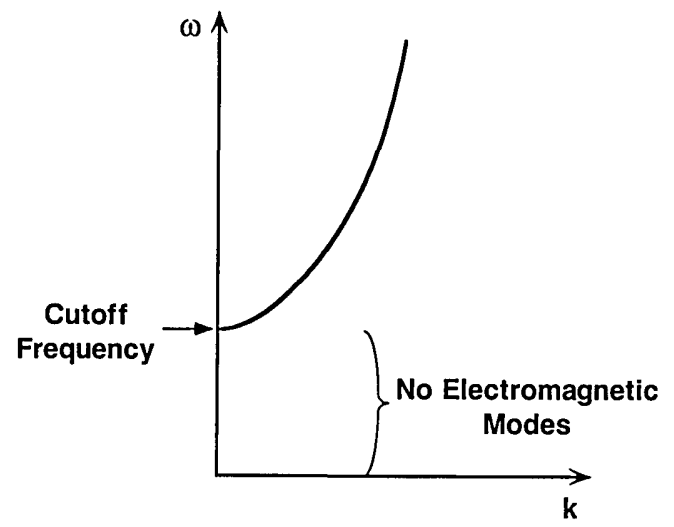

Fig. 2. Electromagnetic wave dispersion between a pair of metal plates. The waveguide dispersion for one of the two polarizations has a cutoff frequency below which no electromagnetic modes and no spontaneous emission are allowed.

emission dates from the Rydberg-atom experiments of Kleppner. A pair of metal plates acts as a waveguide with a cutoff frequency for one of the two polarizations, as shown in Fig. 2. Rydberg atoms are atoms in high-lying principal quantum-number states, which can spontaneously emit in the microwave region of wavelengths. Hulet et al. ${ }^{7}$ shows that Rydberg atoms in a metallic waveguide could be prevented from undergoing spontaneous decay. There were no electromagnetic modes available below the waveguide cutoff.

There is a problem with metallic waveguides, however. They do not scale well into optical frequencies. At high frequencies metals become more and more lossy. These dissipative losses allow for virtual modes, even at frequencies that would normally be forbidden. Therefore it makes sense to consider structures made of positivedielectric-constant materials, such as glasses and insulators, rather than metals. These materials can have low dissipation, even all the way up to optical frequencies. This property is ultimately exemplified by optical fibers, which permit light propagation over many kilometers with negligible losses. Such positive-dielectric-constant materials can have an almost purely real dielectric response with low resistive losses. If these materials are arrayed into a three-dimensionally periodic dielectric structure, a photonic band gap should be possible, employing a purely real, reactive, dielectric response.

The benefits of such a photonic band gap for direct-gap semiconductors are illustrated in Fig. 3. On the right- hand side of Fig. 3 is a plot of the photonic dispersion, (frequency versus wave vector). On the left-hand side of Fig. 3, sharing the same frequency axis, is a plot of the electronic dispersion, showing conduction and valence bands appropriate to a direct-gap semiconductor. Since atomic spacings are 1000 times shorter than optical wavelengths, the electron wave vector must be divided by 1000 to fit on the same graph with the photon wave vectors. The dots in the electron conduction and valence bands are meant to represent electrons and holes, respectively. If an electron were to recombine with a hole, they would produce a photon at the electronic-band-edge energy. As is illustrated in Fig. 3, if a photonic band gap were to straddle the electronic band edge, then the photon produced by electron-hole recombination would have no place to go. The spontaneous radiative recombination of electrons and holes would be inhibited. As can be imagined, this has far-reaching implications for semiconductor photonic devices.

One of the most important applications of spontaneousemission inhibition is likely to be the enhancement of photon-number-state squeezing, which has been playing an increasing role in quantum optics lately. The form of squeezing introduced by Yamamoto ${ }^{3}$ is particularly appealing in that the active element that produces the squeezing effect is none other than the common resistor, as is shown in Fig. 4. When an electrical current flows, it generally carries the noise associated wih the graininess of the electron charge, called shot noise. The corresponding mean-square current fluctuations are

$$
\left\langle(\Delta i)^{2}\right\rangle=2 e i \Delta f,
$$

where $i$ is the average current flow, $e$ is the electronic charge, and $\Delta f$ is the noise bandwidth. While Eq. (2) applies to many types of random physical process, it is far

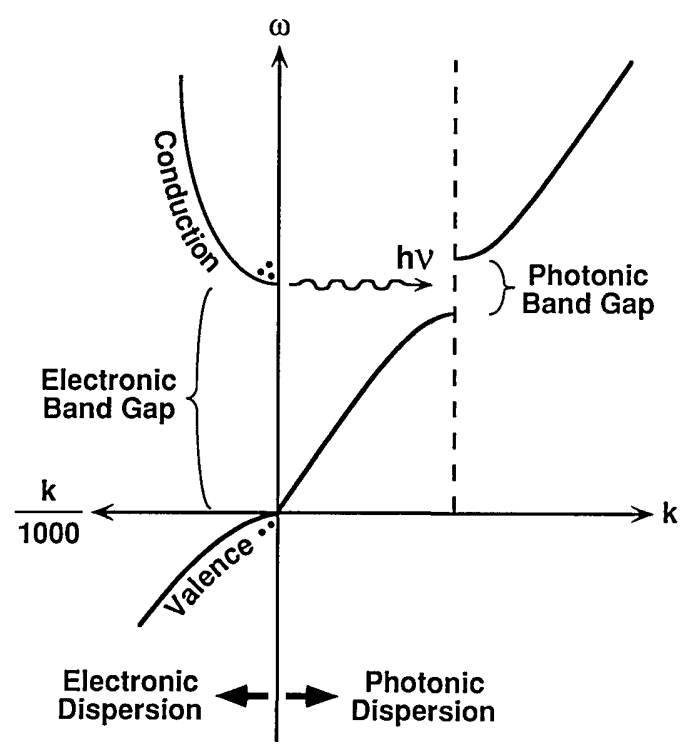

Fig. 3. Right-hand side, the electromagnetic dispersion, with a forbidden gap at the wave vector of the periodicity. Left-hand side, the electron wave dispersion typical of a direct-gap semiconductor; the dots represent electrons and holes. Since the photonic band gap straddles the electronic band edge, electron-hole recombination into photons is inhibited. The photons have no place to go. 


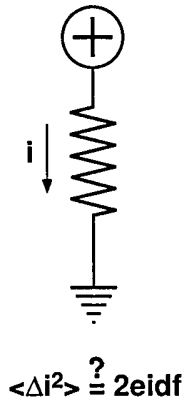

Fig. 4. In a good-quality metallic resistor the current flow is quite regular, producing negligible amounts of shot noise.

from universal. Equation (2) requires that the passage of electrons in the current flow be a random Poissonian process. As early as 1954 Van der Ziel, ${ }^{8}$ in an authoritative book called Noise, pointed out that good-quality metalfilm resistors, when they carry a current, generally exhibit much less noise than predicted by Eq. (2). Apparently the flow of electrons in the Fermi sea of a metallic resistor represents a highly correlated process. This is far from being a random process: the electrons apparently sense one another, producing a level of shot noise far below Eq. (2) (so low as to be difficult to measure and to distinguish from thermal or Johnson noise). Sub-Poissonian shot noise entails the following: Suppose that the average flow consists of 10 electrons per nanosecond. For random flow, the count in successive nanoseconds could be anywhere from 8 to 12 electrons. With good-quality metal-film resistors, the electron count would be 10 for each and every nanosecond.

Yamamoto put this property to good use by driving a high-quantum-efficiency laser diode with such a resistor, as shown in Fig. 5. Suppose that the laser diode quantum efficiency for emission into the cavity mode were $100 \%$. Then for each electron that passes through the resistor there would be one photon emitted into the laser cavity mode. A correlated stream of photons is produced with properties that are unprecedented since the initial exposition of Einstein's photoelectric effect. If the photons are used for optical communication, then a receiver would detect exactly 10 photoelectrons each nanosecond. If 11 photons were detected, then the deviation would be no mere random fluctuation but would represent an intentional signal. Thus information in an optical communications signal could be encoded at the level of individual photons. The name photon-number-state squeezing is associated with the fixed photon number per time interval. Expressed differently, the bit-error rate in optical communication can be diminished by squeezing.

There is a limitation to the squeezing, however. The quantum efficiency for propagation into the lasing mode is not $100 \%$. The $4 \pi$-sr outside the cavity mode can capture a significant amount of random spontaneous emission. If unwanted electromagnetic modes were to capture $50 \%$ of the excitation, then the maximum noise reduction in squeezing would be only $3 \mathrm{~dB}$. Therefore it is necessary to minimize the spontaneous recombination of electrons and holes into modes other than the laser mode. If such random spontaneous events were reduced to $1 \%$, permitting $99 \%$ quantum efficiency into the lasing mode, the corresponding noise reduction would be $20 \mathrm{~dB}$, which is well worth fighting for. Thus we see that control of spontaneous emission is essential for deriving the full benefit from photon-number-state squeezing.

We have advocated the study of photonic band structure for its applications in quantum optics and optical communications. Positive dielectric constants and fully threedimensional forbidden gaps were emphasized. It is now clear that the generality of the concept of the artificial, multidimensional band structure allows for other types of waves, other materials, and various lower dimensional geometries, limited only by imagination and need. Some of these ideas are being presented in other papers in this Journal of the Optical Society of America $B$ feature on photonic band gaps.

\section{SEARCH FOR THE PHOTONIC BAND GAP}

Having decided to create a photonic band gap in three dimensions, we need to settle on a three-dimensionally periodic geometry. For electrons the three-dimensional crystal structures come from nature. Several hundred years of mineralogy and crystallography have classified the naturally occurring three-dimensionally periodic lattices. For photonic band gaps we must create an artificial structure by using our imagination.

The face-centered-cubic (fcc) lattice appears to be favored for photonic band gaps and was suggested independently by $\mathrm{John}^{2}$ and by $\mathrm{me}^{1}$ in our initial proposals. Let us consider the fcc BZ as illustrated in Fig. 6. Various special points on the surface of the BZ are marked. Closest to the center is the $\mathrm{L}$ point, oriented toward the body diagonal of the cube. Farthest away is the $\mathrm{W}$ point, a vertex where four plane waves are degenerate (which will cause problems below). In the cubic directions are the familiar X points.

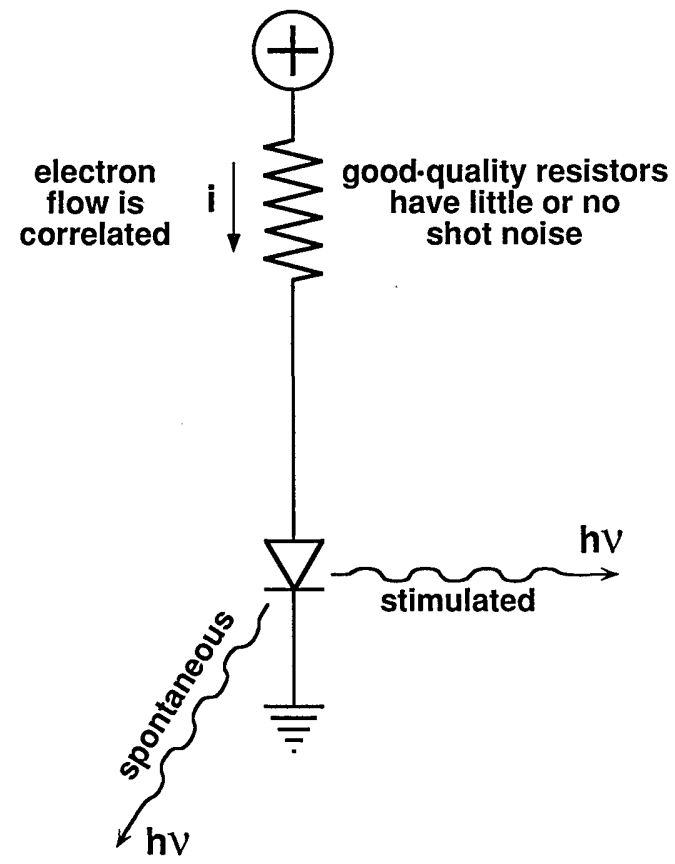

Fig. 5. High-quantum-efficiency laser diode, which converts the correlated flow of electrons from a low-shot-noise resistor into photon-number-state squeezed light. Random spontaneous emission outside the desired cavity mode limits the attainable noise reduction. 


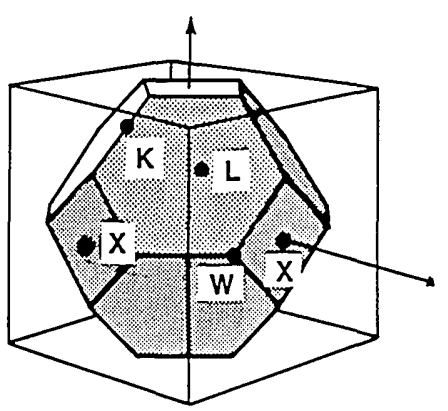

Fig. 6. Fcc BZ in reciprocal space.

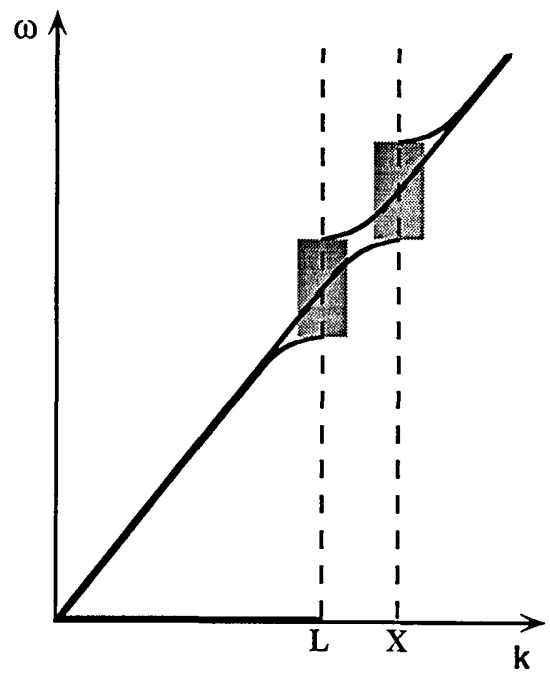

Fig. 7. Forbidden gap (shaded) at the $\mathrm{L}$ point, which is centered at a frequency $\sim 14 \%$ lower than the X-point forbidden gap. Therefore it is difficult to create a forbidden frequency band that overlaps all points along the surface of the BZ.

Consider a plane wave in the $X$ direction. It will sense the periodicity in the cubic direction, forming a standing wave and opening a forbidden gap as indicated by the shading in Fig. 7. Suppose, on the other hand, that the plane wave is going in the $\mathrm{L}$ direction. It will sense the periodicity along the cubic-body diagonal, and a gap will form in that direction as well. But the wave vector to the $L$ point is $\sim 14 \%$ smaller than the wave vector to the $X$ point. Therefore the gap at $\mathrm{L}$ is likely to be centered at a $14 \%$ smaller frequency than the gap at $\mathrm{X}$. If the two gaps are not wide enough, they will not overlap in frequency. In Fig. 7, as shown, the two gaps barely overlap. This is the main problem in achieving a photonic band gap. It is difficult to ensure that a frequency overlap is ensured for all possible directions in reciprocal space.

The lesson from Fig. 7 is that the BZ should most closely resemble a sphere in order to increase the likelihood of a frequency overlap in all directions of space. Therefore let us look at the two common BZ's in Fig. 8, the fcc BZ and the body-centered-cubic (bcc) BZ. The bcc BZ has pointy vertices, which make it difficult for us to achieve a frequency overlap in all directions. Likewise, most other common BZ's deviate even further from a spherical shape. Among all the common BZ's the fcc has the least percentage deviation from a sphere. Therefore, until now all photonic band gaps in three dimensions have been based ${ }^{9}$ on the fcc lattice.
The photonic band gap is different from the idea of a one-dimensional stop band as understood in electrical engineering. Rather, the photonic band gap should be regarded as a stop band with a frequency overlap in all $4 \pi$-sr of space. The earliest antecedent to photonic band structure, dating to 1914 and Sir Lawrence Bragg, ${ }^{10}$ is the dynamic theory of $x$-ray diffraction. Nature gives us fcc crystals, and $\mathrm{x}$-rays are bona fide electromagnetic waves. As early as 1914 narrow stop bands were known to open up. Therefore, what was missing?

The refractive-index contrast for $\mathrm{x}$ rays is tiny, generally 1 part in $10^{4}$. The forbidden $\mathrm{x}$-ray stop bands form extremely narrow rings on the facets of the BZ. As the index contrast is increased, the narrow forbidden rings open up, eventually covering an entire facet of a $B Z$ and ultimately all directions in reciprocal space. We shall see that this requires an index contrast $\gtrsim 2$. The high index contrast is the main new feature of PBS's beyond dynamic $x$ ray diffraction. In addition, we shall see that electromagnetic wave polarization, which is frequently overlooked for $\mathrm{x}$ rays, will play a major role in PBS.

In approaching this subject we adopted an empirical view-point. We decided to make photonic crystals on the scale of microwaves, and then we tested them by using sophisticated coherent microwave instruments. The test setup, shown in Fig. 9, is what we call in optics a Mach-
BCC

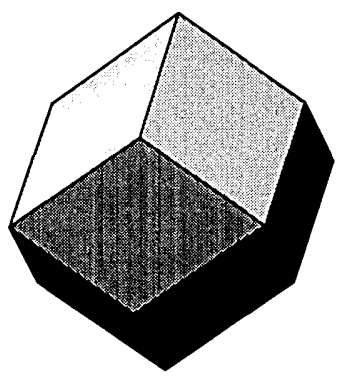

FCC

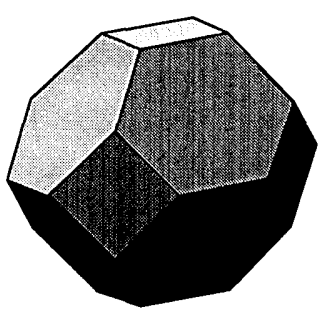

Fig. 8. Two common BZ's for bcc and fcc. The fcc case deviates least from a sphere, favoring a common overlapping band in all directions of space.

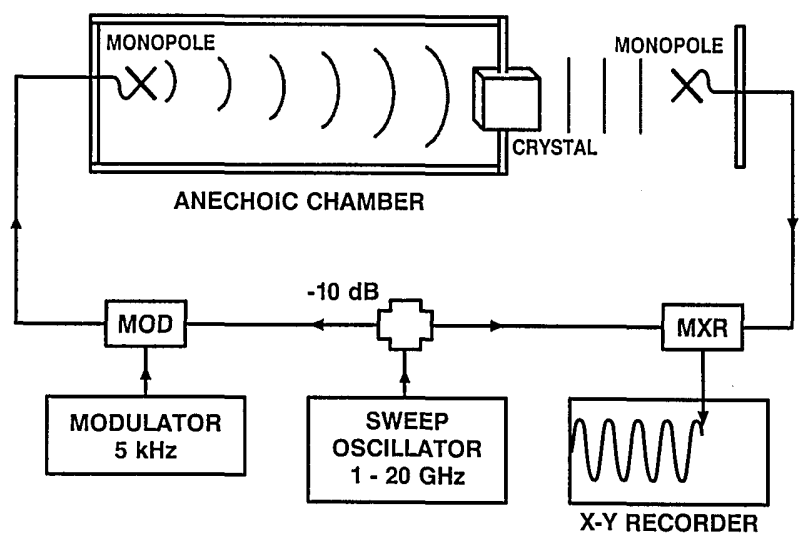

Fig. 9. Homodyne detection system for measuring phase and amplitude in transmission through the photonic crystal under test. A sweep oscillator feeds a $10-\mathrm{dB}$ splitter. Part of the signal is modulated (MOD) and then propagated as a plane wave through the test crystal. The other part of the signal is used as local oscillator for the mixer (MXR) to measure the amplitude change and phase shift in the crystal. Between the mixer and the $X-Y$ recorder is a lock-in amplifier (not shown). 


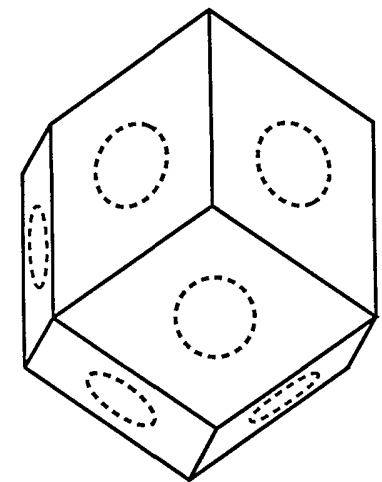

(a)

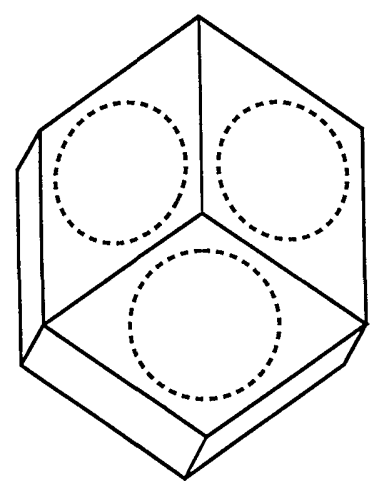

(b)

Fig. 10. WS real-space unit cell of the fcc lattice, a rhombic dodecahedron. (a) Slightly oversized spherical voids are inscribed into the unit cell, breaking through the faces as illustrated. This is the WS cell, corresponding to the photograph in Plate II. (b) WS cell structure with a photonic band gap. Cylindrical holes are drilled through the top three facets of the rhombic dodecahedron and pass through the bottom three facets. The resulting atoms are roughly cylindrical and have a preferred axis in the vertical direction. This WS cell corresponds to the photograph in Plate III.

Zehnder interferometer. It is capable of measuring phase and amplitude in transmission through the microwavescale photonic crystal. In principle one can determine the frequency versus the wave-vector dispersion relations from such coherent measurements. We used a powerful commercial instrument for this purpose, the HP8510 network analyzer. Our approach in the experiments was to measure the forbidden gap in all possible internal directions of reciprocal space. Accordingly the photonic crystal was rotated and the transmission measurements repeated. Because of wave-vector matching along the surface of the photonic crystal, some internal angles could not be reached. To overcome this problem, large microwave prisms, made of polymethyl methacrylate, were placed on either side of the test crystal in Fig. 9.

Early the question arose: Of what material should the photonic crystal be made? The larger the refractive-index contrast, the easier it would be to find a photonic band gap. In optics, however, the largest practical index contrast is that of the common semiconductors $\mathrm{Si}$ and $\mathrm{GaAs}$, with a refractive index $n=3.6$. If that index were inadequate, then photonic crystals would probably never fulfill the goal of being useful in optics. Therefore we decided to restrict the microwave refractive index to 3.6 and the microwave dielectric constant to $n^{2}=12$. A commercial microwave material, Emerson \& Cumming Sycast 12, was particularly suited to the task, since it was machinable with carbide tool bits. Any photonic band structure that was found in this material could simply be scaled down in size and would have identical dispersion relations at optical frequencies and optical wavelengths.

With regard to the geometry of the photonic crystal, there is a universe of possibilities. So far the only restriction that we have made is the choice of fcc lattices. It turns out that a crystal with an fcc BZ in reciprocal space, as shown in Fig. 6, is composed of fcc Wigner-Seitz (WS) unit cells in real space, as shown in Fig. 10. The problem of creating an arbitrary fcc dielectric structure reduces to the problem of filling the fcc WS real-space unit cell with an arbitrary spatial distribution of dielectric material. Real space is then filled by repeated translation and close packing of the WS unit cells. The decision before us is what to put inside the fcc WS cells. There are an infinite number of possible fcc lattices, since anything can be put inside the fundamental repeating unit. The problem: What do we put inside the fec WS unit cell in Fig. 10?

The question provoked strenuous difficulties and false starts over a period of several years before finally being solved. In the first years of this research we were unaware of how difficult the search for a photonic band gap would be. A number of fcc crystal structures were proposed, each representing a different choice for filling the rhombic dodecahedron fcc WS cells in real space. For example, the first suggestion ${ }^{1}$ was to make a threedimensional checkerboard as in Fig. 11, in which cubes were inscribed inside the fcc WS real-space cells in Fig. 10. Later the experiments ${ }^{11}$ adopted spherical "atoms" centered inside the fcc WS cell. Plate I is a photograph of such a structure in which the atoms are precision $\mathrm{Al}_{2} \mathrm{O}_{3}$ spheres, $n \sim 3.06$, each $\sim 6 \mathrm{~mm}$ in diameter. The spheres are supported by a thermal-compression-molded blue foam material of dielectric constant near unity. There are roughly 8000 atoms in Plate I. This structure was tested at a number of filling ratios, from close packing to highly dilute. Nevertheless, it always failed to produce a photonic band gap.

Then we tested the inverse structure, in which spherical voids were inscribed inside the fcc WS real space cell.

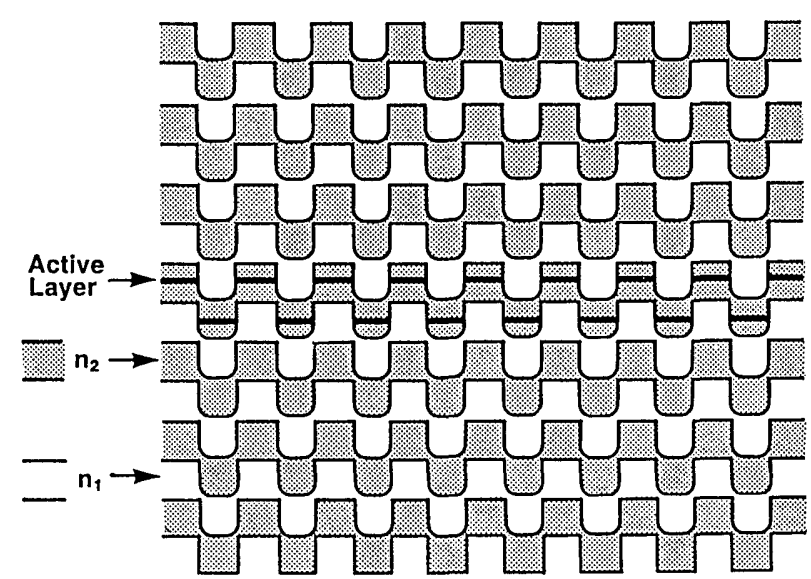

Fig. 11. Fcc crystal, in which the individual WS cells are inscribed with cubes stacked in a three-dimensional checkerboard. 

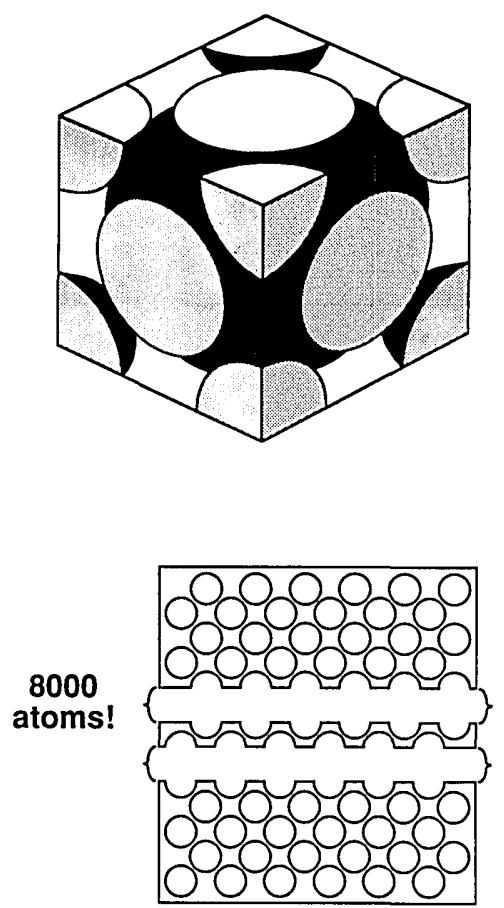

Fig. 12. Construction of fcc crystals, consisting of spherical voids. Hemispherical holes are drilled on both faces of a dielectric sheet. When the sheets are stacked up, the hemispheres meet, producing a fcc crystal.

These could be easily fabricated by drilling hemispheres onto the opposite faces of a dielectric sheet with a spherical drill bit, as shown in Fig. 12. When the sheets were stacked so that the hemispheres faced one another, the result was an fcc array of spherical voids inside a dielectric block. These blocks were also tested over a wide range of filling ratios by progressively increasing the diameter of the hemispheres. These also failed to produce a photonic band gap.

The typical failure mode is illustrated in Fig. 13. As expected, the conduction band at the $L$ point falls at a low frequency, while the valence band at the $W$ point falls at a high frequency. The overlap of the bands at $\mathrm{L}$ and $\mathrm{W}$ results in a band structure that is best described as semimetallic.

The empirical search for a photonic band gap led nowhere until we tested the structure shown in Plate II. This is the spherical-void structure, with oversized voids breaking through the walls of the WS unit cell as shown in Fig. 10(a). For the first time the measurements seemed to indicate a photonic band gap, and we published ${ }^{11}$ the band structure shown in Fig. 14. There appeared to be a narrow gap, centered at $15 \mathrm{GHz}$ and forbidden for both possible polarizations. Unbeknownst to us, however, Fig. 14 harbored a serious error. Instead of a gap at the $\mathrm{W}$ point, the conduction and the valence bands crossed at that point, allowing the bands to touch. This produced a pseudogap with zero density of states but no frequency width. The error arose because of the limited size of the crystal. The construction of crystals with $\sim 10^{4}$ atoms required tens of thousands of holes to be drilled. Such a three-dimensional crystal was still only 12 cubic units wide, limiting the wave-vector resolution and restricting the dynamic range in transmission. Under these conditions it was experimentally difficult to notice a conduction-valence band degeneracy that occurred at an isolated point in $k$ space, such as the $\mathrm{W}$ point.

While we were busy with the empirical search, theorists began serious efforts to calculate the PBS. The most rapid progress was made not by specialists in electromagnetic theory but by electronic-band-structure (EBS) theorists, who were accustomed to solving Schrödinger's equation in three-dimensionally periodic potentials. The early calculations ${ }^{12-15}$ were unsuccessful, however. As a short cut the theorists treated the electromagetic field as a scalar, much as is done for electron waves in Schrödinger's equation. The scalar wave theory of photonic band structure did not agree well with experiment. For example, it predicted photonic band gaps in the dielectric-sphere structure of Plate I, whereas none were observed experimentally. The approximation of Maxwell's equations as a scalar wave equation was not working. Finally, when the full vector Maxwell equations were incorporated, theory began to agree with experiment. Leung ${ }^{16}$ was probably the first to publish a successful vector wave calculation in PBS, followed by others ${ }^{17,18}$ with substantially similar results. The theorists agreed well with one another, and they agreed well with experiment ${ }^{11}$ except at the high-degeneracy points $U$ and particularly W. What the experiment failed to reveal was the degenerate crossing of valence and conduction bands at those points.

The unexpected pseudogap in the crystal of Plate II triggered concern and a search for a way to overcome the problem. A worried editorial ${ }^{19}$ was published in Nature. But even before the editorial appeared, the problem had

\section{$50 \%$ VOLUME FRACTION fCc AIR SPHERES}

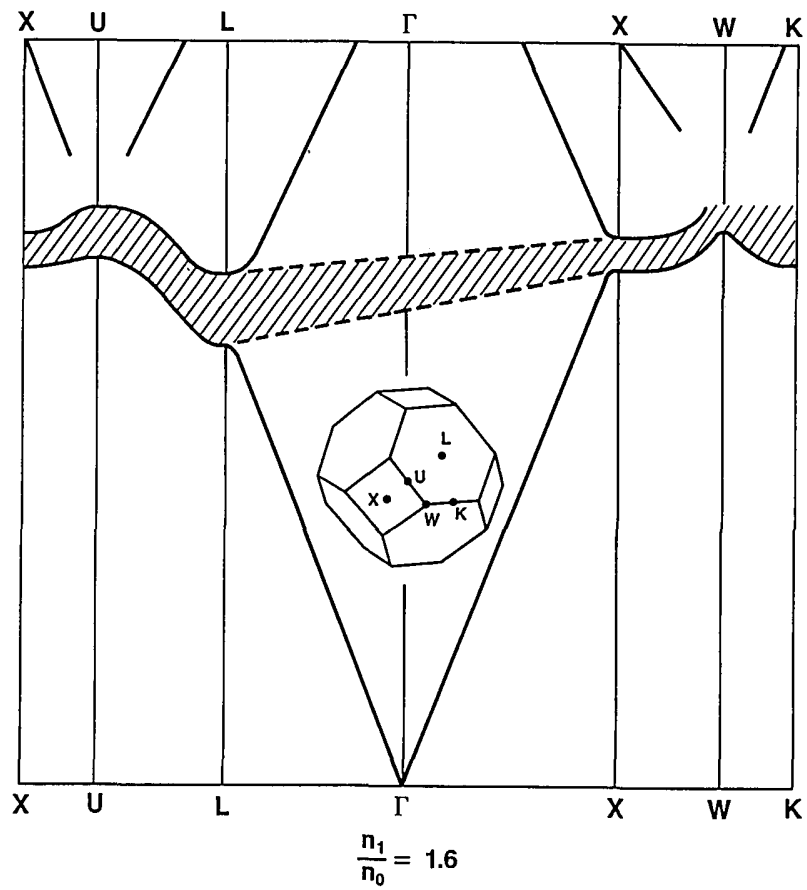

\section{PREDOMINANTLY P POLARIZED}

Fig. 13. Typical semimetallic band structure for a photonic crystal with no photonic band gap. An overlap exists between the conduction band at $\mathrm{L}$ and the valence band at $\mathrm{W}$. 


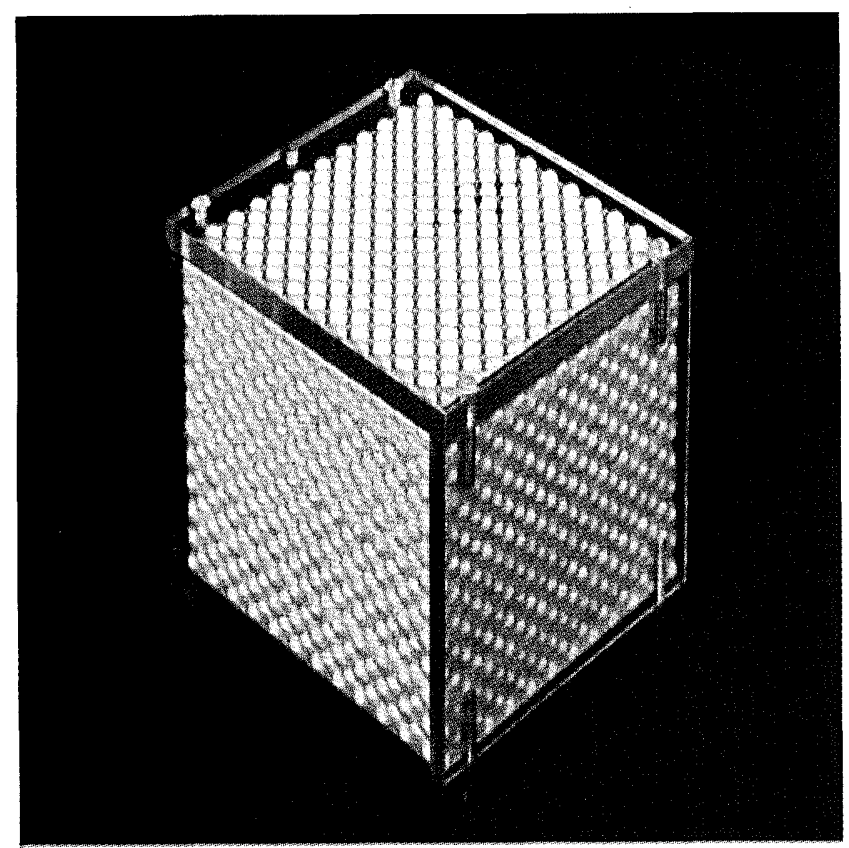

Plate I. Photograph of a three-dimensional fcc crystal consisting of $\mathrm{Al}_{2} \mathrm{O}_{3}$ spheres of refractive index 3.06. The dielectric spheres are supported in place by the blue foam material of refractive index 1.01. These spherical-dielectric-atom structures failed to show a photonic band gap at any volume fraction.

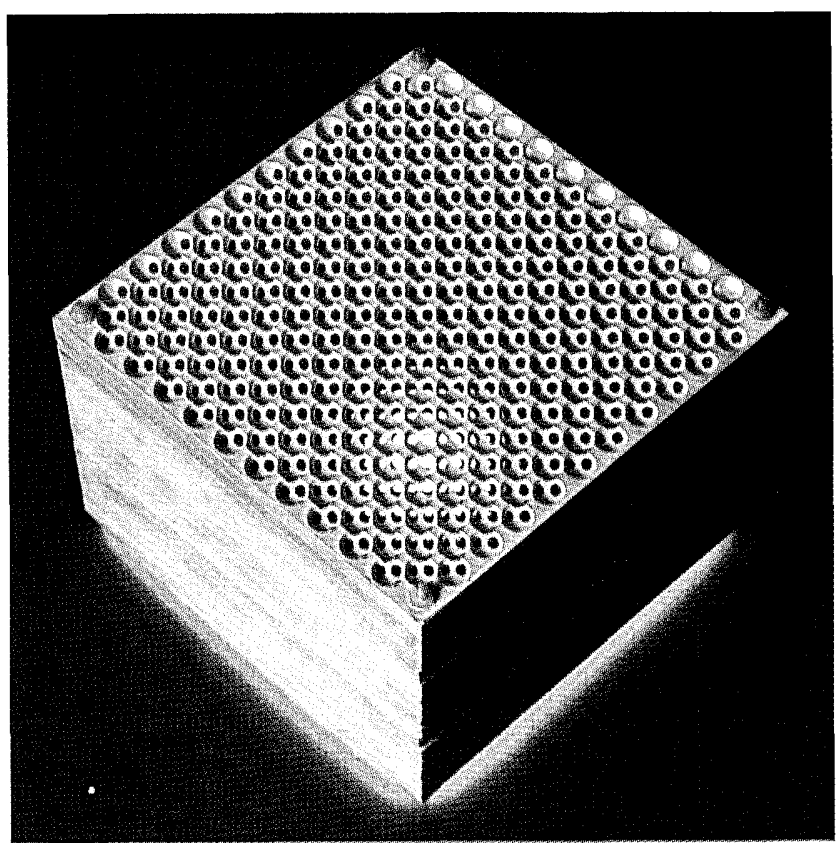

Plate II. Photograph of the photonic crystal corresponding to Fig. 10(a), which had only a pseudogap rather than a full photonic band gap. The spherical voids were closer than close-packed, overlapping and allowing holes to pass through as shown.

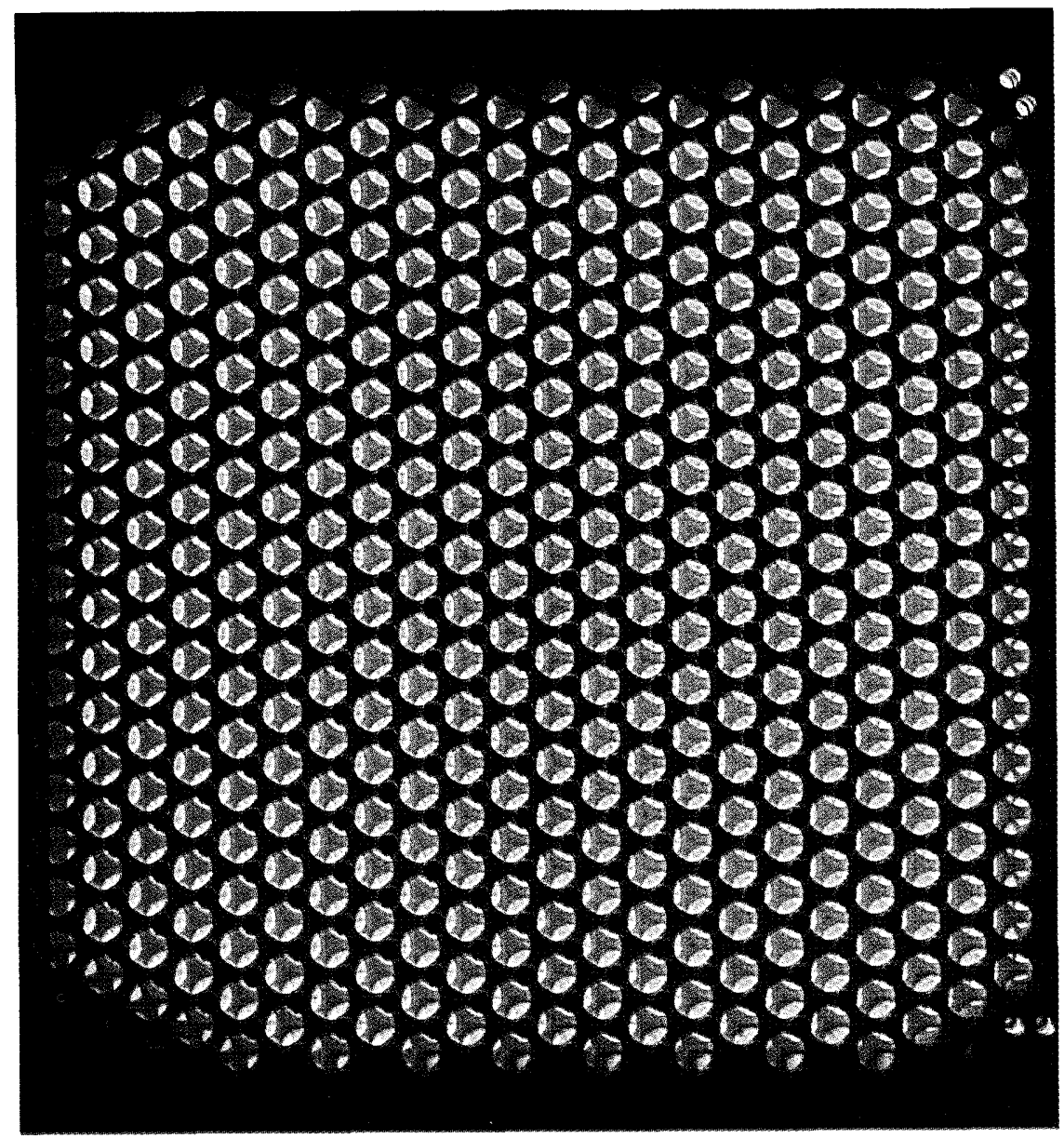

Plate III. Top-view photograph of the nonspherical-atom structure of WS unit cells as shown in Fig. 10(a), constructed by the method of Fig. 15. 



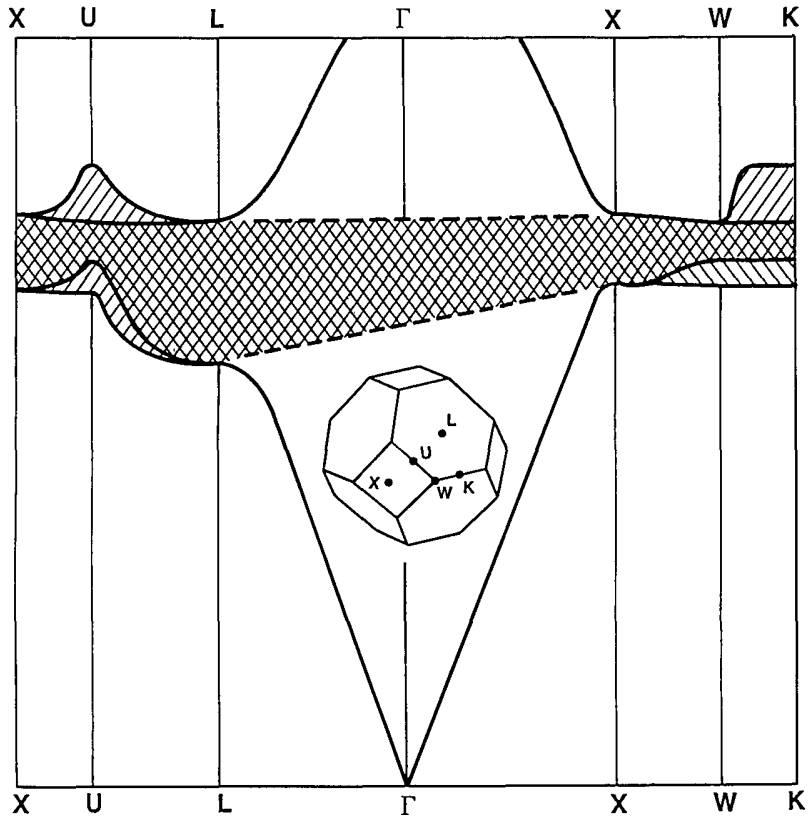

Fig. 14. Purported PBS of the spherical-void structure shown in Figs. 10(a) and Plate II. The rightward-sloping lines represent polarization parallel to the $X$ plane, while the leftward-sloping lines represent the orthogonal polarization, which has a partial component out of the $X$ plane. The cross-hatched region is the reported photonic band gap. This figure fails to show the crossing of the valence and conduction bands at the $\mathrm{W}$ point, which was first discovered by theory. ${ }^{16}$

already been solved by the Iowa State group of Ho et $a l .{ }^{18}$ The degenerate crossing at the $\mathrm{W}$ point was highly susceptible to changes in symmetry of the structure. If one lowered the symmetry by filling the WS unit cell, not by a single spherical atom but by two atoms positioned along the $\langle 111\rangle$ direction, as in the diamond structure, then a full photonic band gap opened up. Their discovery of a photonic band gap in the diamond structure is particularly significant, since the diamond geometry seems to be favored by Maxwell's equations. A form of the diamond structure ${ }^{20}$ gives the widest photonic band gaps, requiring the least index contrast, $n \sim 1.87$.

More generally, one can lower the spherical-void symmetry in Fig. 10(a) by distorting the spheres along the $\langle 111\rangle$ direction, lifting the degeneracy at the $\mathrm{W}$ point. The WS unit cell in Fig. 10(b) has great merit for this purpose. Holes are drilled through the top three facets of the rhombic dodecahedron and exit through the bottom three facets. The beauty of the structure in Fig. 10(b) is that a stacking of WS unit cells results in straight holes that pass through the entire crystal. The atoms are oddshaped, roughly cylindrical voids centered in the WS unit cell with a preferred axis pointing to the top vertex, $\langle 111\rangle$. An operational illustration of the construction that produces an fcc crystal of such WS unit cells is shown in Fig. 15.

A slab of material is covered by a mask that contains a triangular array of holes. Three drilling operations are conducted through each hole, $35.26^{\circ}$ off normal incidence and spread out $120^{\circ}$ on the azimuth. The resulting crisscross of holes below the surface of the slab produces a fully three-dimensionally periodic fcc structure with the WS unit cells given by Fig. 10(b). The drilling can be done by a real drill bit for microwave work or by reactive ion etching to create an fcc structure at optical wavelengths. A top-view photograph of the microwave structure is shown in Plate III.

In spite of the nonspherical atoms in Fig. 10(b), the BZ is identical to the standard fcc BZ shown in textbooks. Nevertheless, we have chosen an unusual perspective from which to view the BZ in Fig. 16. Instead of having the fec $\mathrm{BZ}$ resting on one of its diamond-shaped facets, as is usually done, we have chosen in Fig. 16 to present it resting on a hexagonal face. Since there is a preferred axis for the atoms, the distinctive $\mathrm{L}$ points centered in the top and bottom hexagons are threefold symmetry axes and are labeled $\mathrm{L}_{3}$. The $\mathrm{L}$ points centered in the other six hexagons are symmetric only under a $360^{\circ}$ rotation and are labeled $L_{1}$. It is helpful to know that the $U_{3}$ and $\mathrm{K}_{3}$ points are equivalent, since they are a reciprocal lattice vector apart. Likewise, the $\mathrm{U}_{1}$ and $\mathrm{K}_{1}$ points are equivalent.

Figure 17 shows the dispersion relations along different meridians for our primary experimental sample of normalized hole diameter $d / a=0.469$ and $78 \%$ volume fraction removed (where $a$ is the unit cube length). The ovals represent experimental data with $s$ polarization (perpendicular to the plane of incidence, parallel to the slab surface), while the triangles represent $p$-polarization data (parallel to the plane of incidence, partially perpendicular to the slab surface). The horizontal abscissa in the lower graph of Fig. 17, $\mathrm{L}_{3}-\mathrm{K}_{3}-\mathrm{L}_{1}-\mathrm{U}_{3}-\mathrm{X}-\mathrm{U}_{3}-\mathrm{L}_{3}$, represents a full

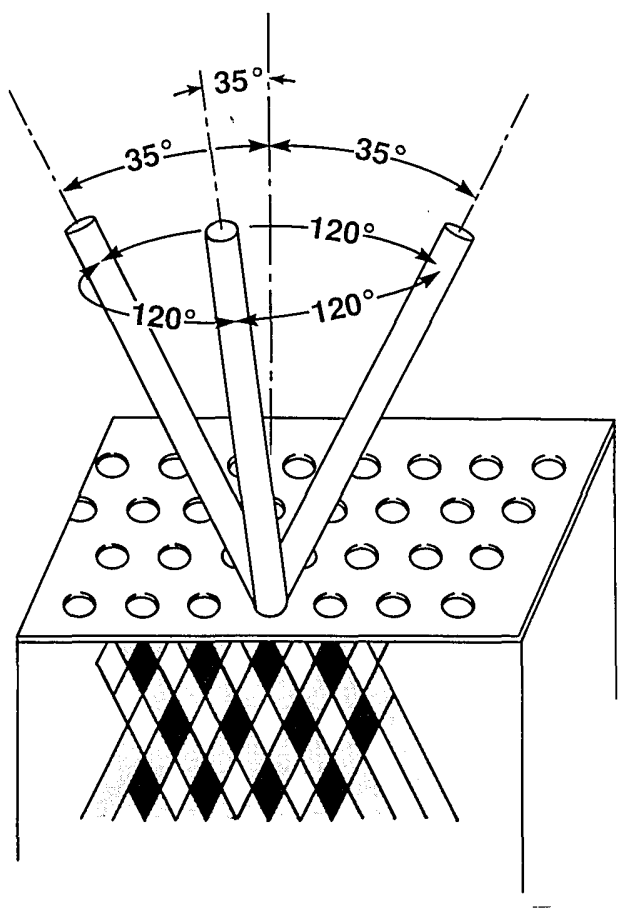

Fig. 15. Method for constructing a fec lattice of the WS cells shown in Fig. 10(b). A slab of material is covered by a mask that consists of a triangular array of holes. Each hole is drilled through three times at an angle $35.26^{\circ}$ away from normal and spread $120^{\circ}$ on the azimuth. The resulting crisscross of holes below the surface of the slab, suggested by the cross-hatching shown here, produces a fully three-dimensionally periodic fcc structure with unit cells as given by Fig. 10(b). The drilling can be done by a real drill bit for microwave work or by reactive ion etching to create fcc structure at optical wavelengths. 


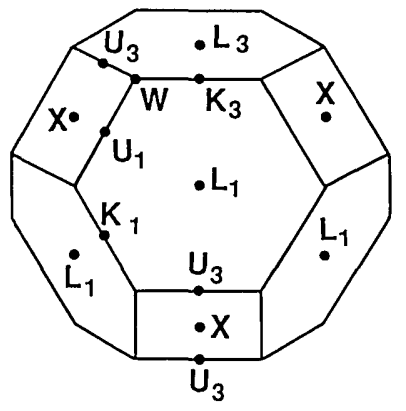

Fig. 16. BZ of a fec structure, incorporating nonspherical atoms as in Fig. 10(b). Since the space lattice is not distorted, this is simply the standard fcc BZ lying on a hexagonal face rather than the usual cubic face. Only the $\mathrm{L}$ points on the top and bottom hexagons are threefold symmetry axes. Therefore they are labeled $L_{3}$. The $L$ points on the other six hexagons are labeled $\mathrm{L}_{1}$. The $\mathrm{U}_{3}$ and $\mathrm{K}_{3}$ points are equivalent, since they are a reciprocal lattice vector apart. Likewise, the $\mathrm{U}_{1}$ and $\mathrm{K}_{1}$ points are equivalent.
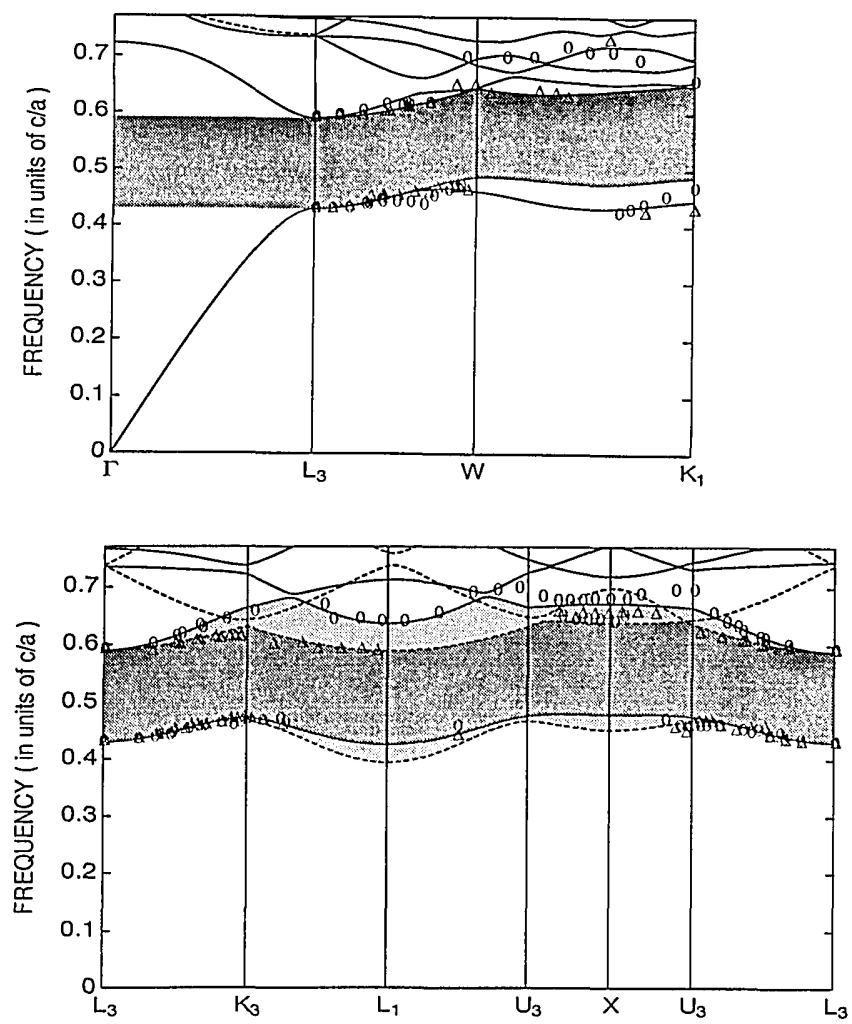

Fig. 17. Frequency versus wave vector ( $\omega$ versus $\mathbf{k}$ ) dispersion along the surface of the $\mathrm{BZ}$ shown in Fig. 16, where $c / a$ is the speed of light divided by the fcc cube length. The ovals and the triangles are the experimental points for $s$ and $p$ polarization, respectively. The solid and dashed curves are the calculations for $s$ and $p$ polarization, respectively. The dark shaded band is the totally forbidden band gap. The lighter shaded stripes above and below the dark band are forbidden only for $s$ and $p$ polarization, respectively.

meridian from the north pole to the south pole of the BZ. Along this meridian the Bloch wave functions separate nearly into $s$ and $p$ polarizations. The $s$ - and $p$-polarized theory curves are the solid and dashed curves, respectively. The dark shaded band is the totally forbidden photonic band gap. The lighter shaded stripes above and below the dark band are forbidden only for $s$ and $p$ polarization, respectively.
At a typical semiconductor refractive index, $n=3.6$, the three-dimensional forbidden gap width is $19 \%$ of its center frequency. Calculations ${ }^{21}$ indicate that the gap remains open for refractive indices as low as $n=2.1$ for circular holes as in Fig. 15. We have also measured the imaginary wave-vector dispersion within the forbidden gap. At midgap we find an attenuation of $10 \mathrm{~dB}$ per unit cube length $a$. Therefore the photonic crystal need not be many layers thick to expel the zero-point electromagnetic field effectively. The construction of Fig. 15 can be implemented by reactive ion etching, as shown in Fig. 18. In reactive ion etching, the projection of circular mask openings at $35^{\circ}$ leaves oval holes in the material, which might not perform as well. Fortunately it was found ${ }^{21}$ in defiance of Murphy's law, that the forbidden gap width for oval holes is actually improved by fully $21.7 \%$ of its center frequency.

\section{DOPING THE PHOTONIC CRYSTAL}

The perfect semiconductor crystal is quite elegant and beautiful, but it becomes ever more useful when it is doped. Likewise the perfect photonic crystal can become of even greater value when a defect ${ }^{22}$ is introduced.

Lasers, for example, require that the perfect threedimensional translational symmetry be broken. Even though spontaneous emission from all $4 \pi$ sr should be inhibited, a local electromagnetic mode, linked to a defect, to accept the stimulated emission is still necessary. In one-dimensional distributed-feedback lasers ${ }^{23}$ a quarter-wavelength defect is introduced, effectively forming a Fabry-Perot cavity as shown in Fig. 19. In threedimensional PBS a local defect-induced structure resembles a Fabry-Perot cavity, except that it reflects radiation back upon itself in all $4 \pi$ spatial directions.

The perfect three-dimensional translational symmetry of a dielectric structure can be altered in one of two ways. (1) Extra dielectric material may be added to one of the unit cells. We find that such a defect behaves much like a donor atom in a semiconductor. It gives rise to donor modes, which have their origin at the bottom of the conduction band. (2) Conversely, translational symmetry

\section{microfabrication by reactive ions}
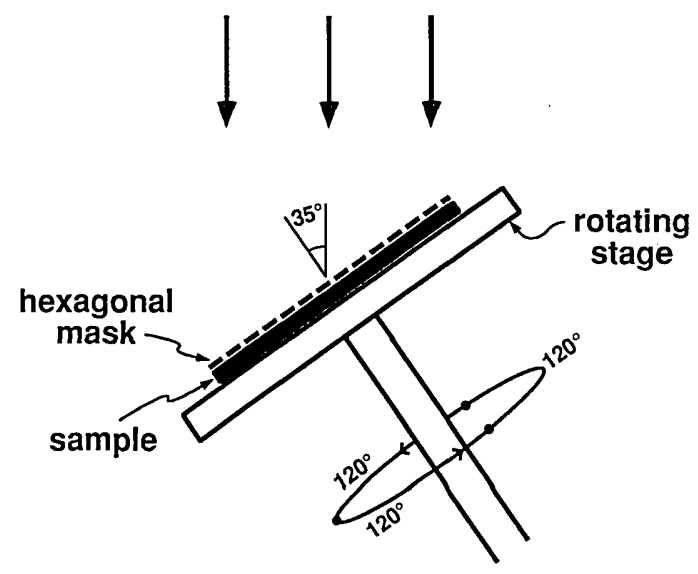

Fig. 18. Construction of the nonspherical-void photonic crystal of Figs. 10(b) and 15-17 and of Plate III by reactive ion etching. 


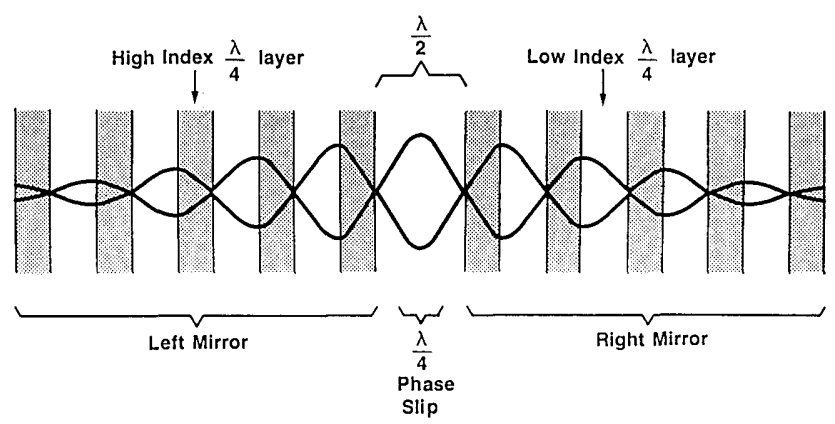

Fig. 19. One-dimensional Fabry-Perot resonator, made of multilayer dielectric mirrors with a space of one half-wavelength between the left- and right-hand mirrors. The net effect is to introduce a quarter-wavelength phase slip defect into the overall periodic structure. A defect mode is introduced at midgap.

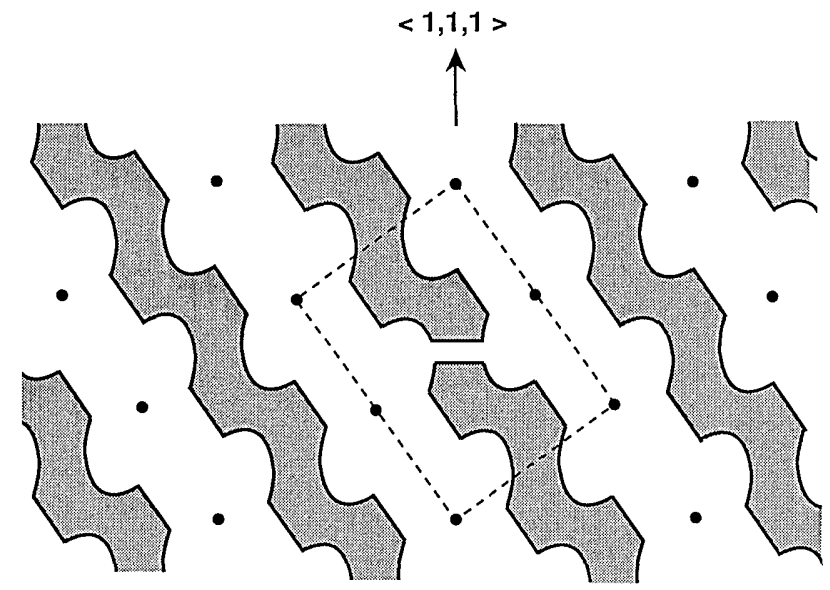

Fig. 20. $\langle 1, \overline{1}, 0\rangle$ Cross-sectional view of our fcc photonic crystal, consisting of nonspherical air atoms centered on the large dots. Dielectric material is represented by the shaded areas. The dashed rectangle is a face-diagonal cross section of the unit cube. Donor defects consisted of a dielectric sphere centered on an atom. We selected an acceptor defect as shown centered in the unit cube. It consists of a missing horizontal slice in a single vertical rib.

can be broken by removal of some dielectric material from one of the unit cells. Such defects resemble acceptor atoms in semiconductors. The associated acceptor modes have their origin at the top of the valence band. We shall find that acceptor modes are particularly well suited to act as laser microresonator cavities. Indeed, it appears that photonic crystals made of sapphire or other low-loss dielectrics will make the highest- $Q$ single-mode cavities (of modal volume $\sim 1 \lambda^{3}$ ), covering electromagnetic frequencies above the useful working range of superconducting metallic cavities. The short-wavelength limit in the ultraviolet is set by the availability of optical materials with refractive index $\geqslant 2$, the threshold index ${ }^{18,21}$ for the existence of a photonic band gap.

Figure 20 is a $\langle 1, \overline{1}, 0\rangle$ cross section of our photonic crystal of Fig. 10(b) of Figs. 15-17, and of Plate III, shown as a cut through the center of a unit cube. Shading represents dielectric material. The large dots are centered on the air atoms, and the rectangular dashed line is a face-diagonal cross section of the unit cube. Since we could design the structure at will, donor defects were chosen to consist of a single dielectric sphere centered in an air atom. Likewise, by breaking one of the interconnecting ribs, it is easy to create acceptor modes. We selected an acceptor defect, as shown in Fig. 20, centered in the unit cube. It consists of a vertical rib that has a missing horizontal slice.

The heart of our experimental apparatus is a photonic crystal embedded in microwave absorbing pads as shown in Fig. 21. The photonic crystals were 8-10 atomic layers thick in the $\langle 1,1,1\rangle$ direction. Monopole antennas, consisting of $6-\mathrm{mm}$ pins, coupled radiation to the defect mode. The HP 8510 network analyzer was set up to measure transmission between the antennas. Figure 22(a) shows the transmission amplitude in the absence of a defect. There is very strong attenuation $\left(\sim 10^{-5}\right)$ between 13 and $16 \mathrm{GHz}$, marking the valence- and conductionband edges of the forbidden gap. This is a tribute to both the dynamic range of the network analyzer and the sizable imaginary wave vector in the forbidden gap.

A transmission spectrum in the presence of an acceptor defect is shown in Fig. 22(b). Most of the spectrum is unaffected, except at the electromagnetic frequency marked deep acceptor within the forbidden gap. At that precise frequency, radiation hops from the transmitting antenna to the acceptor mode and then to the receiving antenna. The acceptor-level frequency, within the forbidden gap, is dependent on the volume of material removed. Figure 23 shows the acceptor-level frequency as a function of the defect volume removed from one unit cell. When a relatively large volume of material is removed, the acceptor level is deep, as is shown in Fig. 22(b). A smaller amount of material removed results in a shallow acceptor level nearer the valence band. If the removed material volume falls below a threshold volume, the accep-

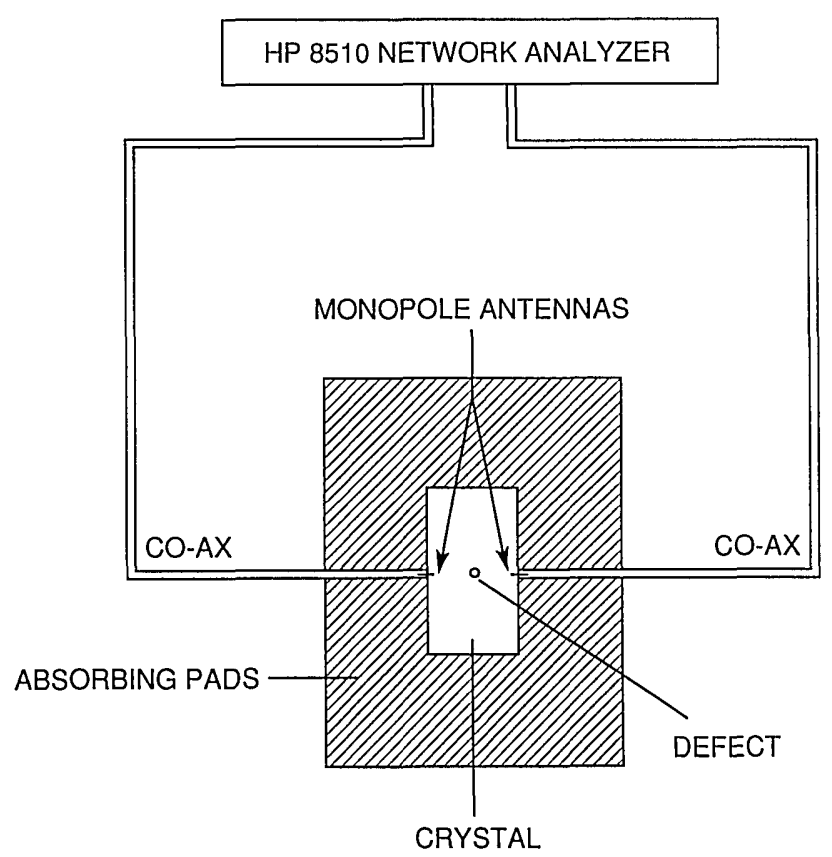

Fig. 21. Experimental configuration for the detection of local electromagnetic modes in the vicinity of a lattice defect. Transmission amplitude attenuation from one antenna to the other is measured. At the local mode frequency the signal hops by means of the local mode in the center of the photonic crystal, producing a local transmission peak. The signal propagates in the $\langle 1,1,1\rangle \mathrm{di}-$ rection through 8-10 atomic layers. Co-Ax, coaxial line. 

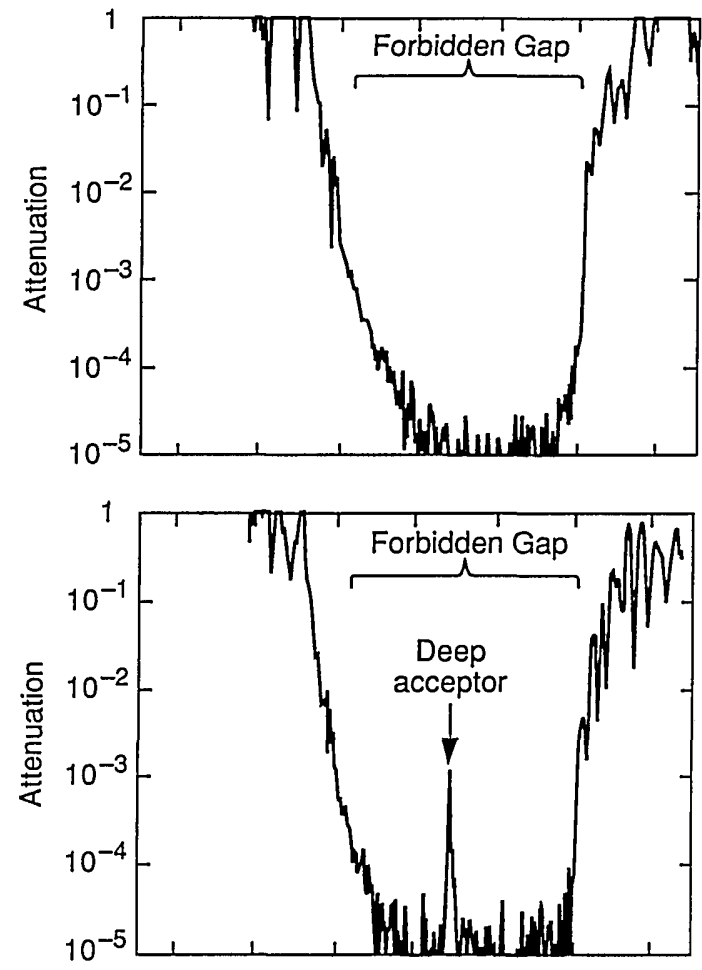

(b)

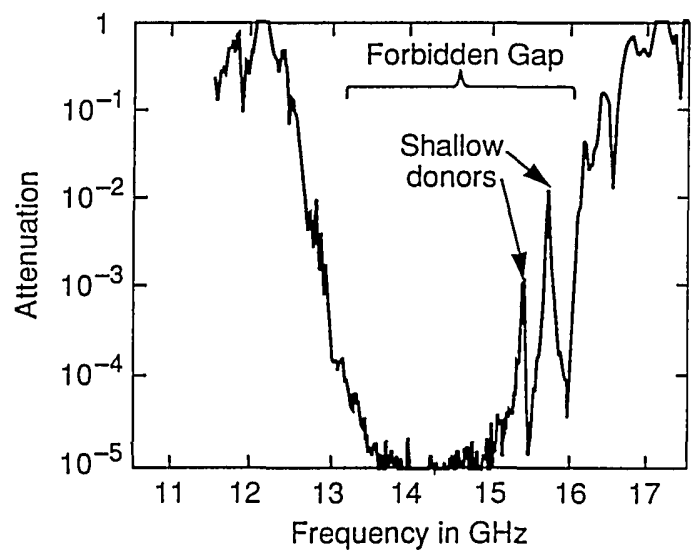

Fig. 22. (a) Transmission attenuation through a defect-free photonic crystal as a function of microwave frequency. The forbidden gap falls between 13 and $16 \mathrm{GHz}$. (b) Attenuation through a photonic crystal with a single acceptor in the center. The relatively large acceptor defect volume shifted its frequency to near midgap. The electromagnetic resonator $Q$ was $\sim 1000$, limited only by the loss tangent of the dielectric material. (c) Attenuation through a photonic crystal with a single donor defect, an off-center dielectric sphere, leading to two shallow donor modes.

tor level falls within the continuum of levels below the top of the valence band, becoming metastable.

On an expanded frequency scale we can measure the resonator $Q$ of the deep acceptor mode, which is $Q \sim 1000$, as limited by the loss tangent of the Emerson \& Cumming Stycast material of which the photonic crystal was made.

The behavior of an off-center donor defect is shown in Fig. 22(c). In this case the donor volume was only slightly above the required threshold for forming bound donor modes. Already two shallow donor modes can be seen in Fig. 22(c). When the donor is centered in the WS unit cell, the two modes merge to form a doubly degenerate donor level as in Fig. 23. Single donor defects seem to produce multiple donor levels. Figure 23 gives the donorlevel frequency as a function of donor volume. As in the case of acceptors, there is a threshold defect volume required for the creation of bound modes below the conduction-band edge. However, the threshold volume for donor defects is almost 10 times larger than the acceptor threshold volume. Apparently this difference is due to the electric-field concentration in the dielectric ribs at the top of the valence band. Bloch wave functions at the top of the valence band are rather easily disrupted by the missing rib segment.

We have chosen in Fig. 23 to normalize the defect volume to a natural volume of the physical system, $(\lambda / 2 n)^{3}$, which is basically a cubic half-wavelength in the dielectric medium. More specifically, $\lambda$ is the vacuum wavelength at the midgap frequency and $n$ is the refractive index of the dielectric medium. Since we are measuring a dielectric volume, it makes sense to normalize to a halfwavelength cube as measured at the dielectric refractive index. Based on the reasonable scaling of Fig. 23, our choice of volume normalization would seem justified.

The vertical rib with a missing horizontal slice, shown in Fig. 20, can be readily microfabricated. It should be possible to create it in III-V materials by growing an Alrich epitaxial layer and lithographically patterning it down to a single dot that is the size of one of the vertical ribs. After regrowth of the original III-V composition and reactive ion etching of the photonic crystal, HF acid etching $^{24}$ with a selectivity $\geq 10^{8}$ will be used to remove the Al-rich horizontal slice from the one rib that contains such a layer. The resonant frequency of the microcavity can be controlled by the thickness of the Al-rich sacrificial layer.

Therefore, by doping the photonic crystal, it is possible to create high- $Q$ electromagnetic cavities whose modal volume is less than a half-wavelength cubed. These doped photonic crystals would be similar to metallic microwave cavities, except that they would be usable at higher frequencies, where metal cavity walls would become lossy. With sapphire as a dielectric, for example, it should be possible to make a millimeter-wave cavity with $Q \geqslant 10^{9}$. The idea is not to compete directly with superconducting cavities but rather to operate a higher frequencies, where the superconductors become lossy. Given the requirement for a refractive index $>2$, doped photonic crystals should work well up to ultraviolet wavelengths at which diamond crystals and $\mathrm{TiO}_{2}$ are still transparent.

\section{APPLICATIONS}

The forthcoming availability of single-mode microcavities at optical frequencies will lead to a new situation in quantum electronics. Of course microwave cavities that contain a single electromagnetic mode have been known for a long time. At microwave frequencies, however, spontaneous emission of electromagnetic radiation is a weak and unimportant process. At optical frequencies spontaneous emission comes into its own. Now we can combine the physics and technology of spontaneous emission with the capability for single-mode microcavities at optical frequencies, where spontaneous emission is important. This combination is fundamentally a new regime in quantum electronics. 


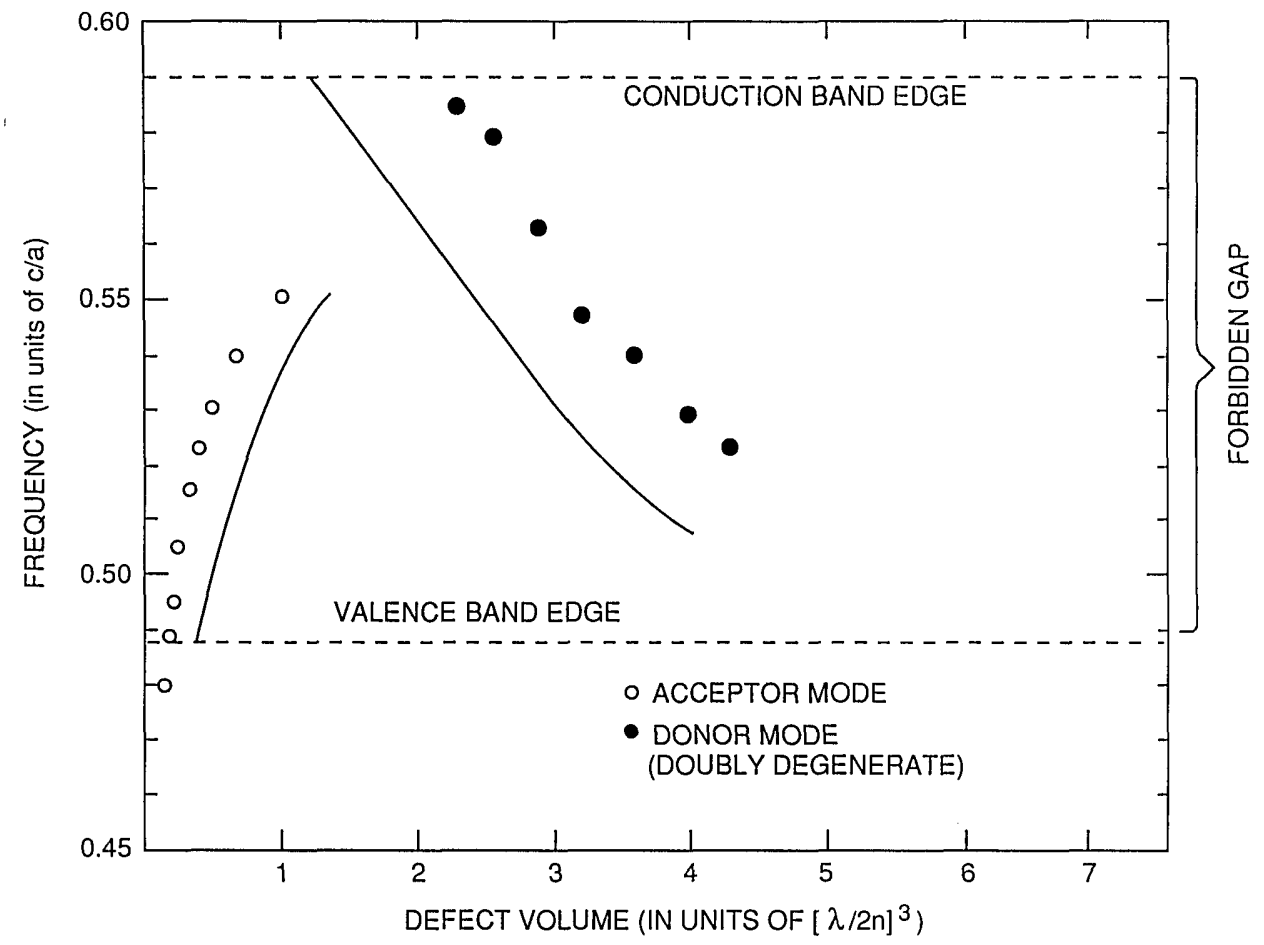

Fig. 23. Donor and acceptor mode frequencies as a function of normalized donor and acceptor defect volume. The points are experimental, and the corresponding curves are calculated. Defect volume is normalized to $(\lambda / 2 n)^{3}$, where $\lambda$ is the midgap vacuum wavelength and $n$ is the refractive index. A finite defect volume is necessary to bind a mode in the forbidden gap.

The major example of this new type of device is the single-mode light-emitting diode (SM-LED), which can have many of the favorable coherence properties of lasers while being a more reliable and thresholdless device. Progress in electromagnetic microcavities permits all the spontaneous emission of an LED to be funneled into a single electromagnetic mode.

As the interest in the low-threshold semiconductor laser diode has grown, e.g., for optical interconnects, its spontaneously luminescent half-brother, the LED, has begun to reemerge in a new form. In this new form the LED is surrounded by an optical cavity. The idea is for the optical cavity to make available only a single electromagnetic mode for the output spontaneous emission from the semiconductor diode. In fact the figure of merit for such a cavity is $\beta$, the fraction of spontaneous emission that is funneled into the desired mode. What is new for this application is the prospective ability to make high $-\beta$ cavities at optical frequencies that employ photonic crystals. The three-dimensional character of the cavities ensures that spontaneous emission will not seek out those neglected modes that are found propagating in a direction where this is no optical confinement.

With all the spontaneous emission funneled into a single optical mode, the SM-LED can begin to have many of the coherence and statistical properties normally associated with above-threshold lasing. The essential point is that the spontaneous-emission factor $\beta$ should approach unity. (A closely related concept is that of the zero-threshold laser, in which the high-spontaneous-emission factor produces a soft and indistinct threshold characteristic in the curve of the light output versus the current input for laser diodes.) The idea is to combine the advantages of the LED, which is thresholdless and highly reliable, with those of the semiconductor laser, which is coherent and efficient.

The coherence properties of the SM-LED are illustrated in Fig. 24. In a laser single-mode emission is the result of gain saturation and mode competition. In the SM-LED there is no gain and therefore no gain saturation, but the

\section{Single-Mode Light-Emitting Diode}

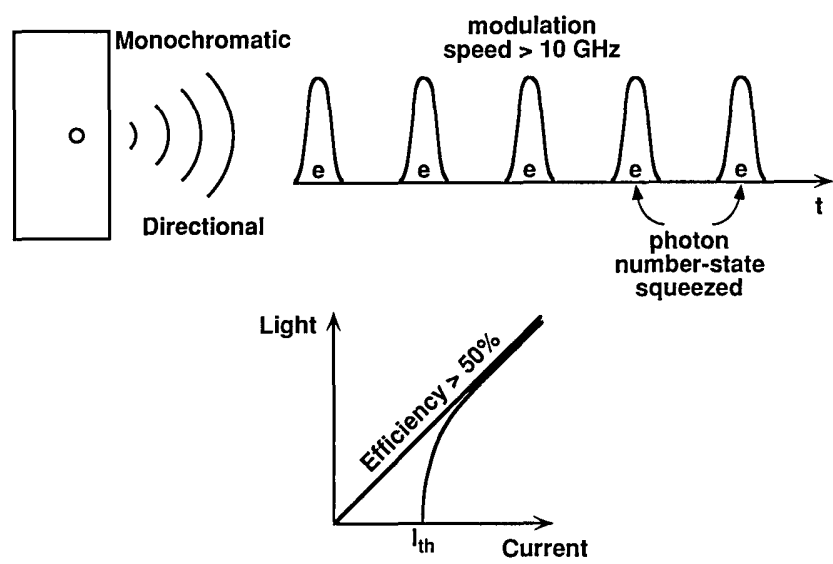

Fig. 24. Properties of the SM-LED, whose cavity is represented by the small circle inside the rectangular photonic crystal at left. The words Monochromatic and Directional represent the temporal and spatial coherence of the SM-LED output, as is explained in the text. The modulation speed can be $>10 \mathrm{GHz}$, and the differential quantum efficiency can be $>50 \%$, which is competitive with that of laser diodes. But there is no threshold current for the SM-LED, as indicated by the curves for light output versus the input current at the bottom. The regular stream of photoelectrons, e's, is meant to represent photon-numberstate squeezing, which can be produced by the SM-LED if the spontaneous-emission factor $\beta$ of the cavity is high enough. 
Table 1. Summary of Differences and Similarities between Photonic and Electronic Band Structures

\begin{tabular}{lcc}
\hline Characteristic & EBS & PBS \\
\hline $\begin{array}{l}\text { Underlying } \\
\text { dispersion } \\
\text { relation }\end{array}$ & Parabolic & Linear \\
$\begin{array}{l}\text { Angular } \\
\text { Momentum }\end{array}$ & $\begin{array}{l}\text { Spin 1/2 } \\
\text { scalar wave } \\
\text { approximation }\end{array}$ & $\begin{array}{c}\text { Spin 1 } \\
\text { vector wave } \\
\text { character }\end{array}$ \\
$\begin{array}{l}\text { Accuracy } \\
\text { of band } \\
\text { theory }\end{array}$ & $\begin{array}{l}\text { Approximate } \\
\text { owing to } \\
\text { electron-electron }\end{array}$ & $\begin{array}{c}\text { Essentially } \\
\text { exact }\end{array}$ \\
\hline
\end{tabular}

output is still single mode because only one mode is available for emission. Since a single spatial mode can always be mode converted into a plane wave, the SM-LED can be regarded as having spatial coherence.

What about temporal coherence? The spectral linewidth of the SM-LED is narrower than the luminescence band of the semiconductor. All the radiation is funneled into the narrow spectral band determined by the microcavity $Q$. Thus SM-LED's have both spatial and temporal coherence, as represented by the words Directional and Monochromatic in Fig. 24.

What about the modulation speed of SM-LED's in comparison with that of laser diodes under direct-current modulation? Generally, the modulation speed depends on the carrier lifetime. Since electron-hole pairs in laser diodes experience both spontaneous and stimulated recombination, they have an advantage. However, single-mode cavities concentrate zero-point electric-field fluctuations into a smaller volume, creating a stronger matrix element for spontaneous emission. Detailed calculations indicate that spontaneous emission can be sped up by a factor of $\sim 10$ owing to this cavity quantum-electrodynamic (QED) effect. In Fig. 24 we indicate that a modulation speed $>10 \mathrm{GHz}$ should be possible for SM-LED's.

The same cavity QED effects can enhance the spontaneous-emission efficiency of SM-LED's, since the radiative rate can then compete more successfully with nonradiative rates. External efficiency should exceed 50\%, but this can come most easily from intelligent LED design ${ }^{25}$ rather than from cavity QED effects.

Plotted at the bottom of Fig. 24 is the light output versus the input current of SM-LED's and laser diodes. SM-LED's can compete with laser diodes in terms of differential external efficiency, but the SM-LED's can have the advantage of not demanding any threshold current. Lack of threshold behavior makes the output power and the operating wavelength of an SM-LED relatively insensitive to ambient temperature. Combined with the inherent reliability of an LED, this should produce many system advantages for the SM-LED concept.

The final SM-LED property illustrated in Fig. 24 is photon-number-state squeezing, as suggested by the regular sequence of photoelectrons on the horizontal line. Stimulated emission is not required for those exotic squeezing effects. The critical variable is absolute quantum efficiency. If the quantum efficiency of the SM-LED is high, then these squeezing correlations will exist in the spontaneous output of the single-mode LED. This re- quires, most of all, a high spontaneous-emission factor $\beta$, our overall figure of merit for microcavities.

In the feature on photonic band gaps in this journal issue there are many other applications given for photonic crystals, particularly in the microwave and millimeterwave regimes. The applications are highly imaginative, and they have gone far beyond our initial goals for using photonic crystals in quantum optics.

\section{CONCLUSIONS}

It is worthwhile to summarize the similarities and the differences between photonic and electronic band structure. This is best done by reference to Table 1 .

Electrons are massive, and so the underlying dispersion relation for electrons in crystals is parabolic. Photons have no mass, so the underlying dispersion relation is linear. But as a result of the periodicity the photons develop an effective mass in PBS, and this should come as little surprise.

Electrons have spin $1 / 2$, but frequently this spin is ignored, and Schrödinger's equation is treated in a scalar wave approximation. In electronic band theory the spin $1 / 2$ is occasionally important, however. In contrast, photons have spin 1, but it is generally never a good approximation to neglect polarization in PBS calculations.

Finally, we come to the accuracy of band theory. It is sometimes believed that band theory is always a good approximation in electronic structure. This is not really true. When there are strong correlations, as in the high$T_{c}$ superconductors, band theory is not even a good zerothorder approximation. Photons are highly noninteracting, so, if anything, band theory makes more sense for photons than for electrons.

The final point to make about photonic crystals is that they are nearly empty structures, consisting of $\sim 78 \%$ empty space. But in a sense they are much emptier than that. They are emptier and quieter than even the vacuum, since they contain not even zero-point fluctuations within the forbidden frequency band.

\section{ACKNOWLEDGMENTS}

I thank John Gural for drilling all the holes and Tom Gmitter for making the measurements. Thanks are also due to Ming Leung and Bob Meade for their collaborative work. The Iowa State group is thanked for making me aware of its diamond structure results before publication.

*Work performed at Bell Communications Research, Navesink Research Center, Red Bank, New Jersey 077017040.

\section{REFERENCES AND NOTES}

1. E. Yablonovitch, Phys. Rev. Lett. 58, 2059 (1987).

2. S. John, Phys. Rev. Lett. 58, 2486 (1987).

3. Y. Yamamoto, S. Machida, and W. H. Richardson, Science 255, 1219 (1992).

4. E. M. Purcell, Phys. Rev. 69, 681 (1946).

5. K. H. Drexhage, in Progress in Optics 12, E. Wolf, ed. (NorthHolland, Amsterdam, 1974), p. 165.

6. V. P. Bykov, Sov. J. Quantum Elec. 4, 861 (1975). 
7. R. G. Hulet, E. S. Hilfer, and D. Kleppner, Phys. Rev. Lett. 55, 2137 (1985).

8. A. Van der Ziel, Noise (Prentice-Hall, New York, 1954).

9. There have been some reports recently of a photonic band gap in a simple cubic geometry. H. S. Sozuer, J. W. Haus, and R. Inguva, Phys. Rev. B 45, 13, 962 (1992).

10. C. G. Darwin, Philos. Mag. 27, 675 (1914).

11. E. Yablonovitch and T. J. Gmitter, Phys. Rev. Lett. 63, 1950 (1989).

12. S. Satpathy, Z. Zhang, and M. R. Salehpour, Phys. Rev. Lett. 64, 1239 (1990).

13. K. M. Leung and Y. F. Liu, Phys. Rev. B 41, 10188 (1990).

14. S. John and R. Rangarajan, Phys. Rev. B 38, 10101 (1988).

15. E. N. Economu and A. Zdetsis, Phys. Rev. B 40, 1334 (1989).

16. K. M. Leung and Y. F. Liu, Phys. Rev. Lett. 65, 2646 (1990).

17. Z. Zhang and S. Satpathy, Phys. Rev. Lett. 65, 2650 (1990).

18. K. M. Ho, C. T. Chan, and C. M. Soukoulis, Phys. Rev. Lett. 65, 3152 (1990).
19. J. Maddox, Nature (London) 348, 481 (1990).

20. C. T. Chan, K. M. Ho, and C. M. Soukoulis, Europhys. Lett. 16, 563 (1991).

21. E. Yablonovitch, T. J. Gmitter, and K. M. Leung, Phys. Rev. Lett. 67, 2295 (1991)

22. E. Yablonovitch, T. J. Gmitter, R. D. Meade, A. M. Rappe, K. D. Brommer, and J. D. Joannopoulos, Phys. Rev. Lett. 67, 3380 (1991).

23. H. A. Haus and C. V. Shank, IEEE J. Quantum Electron. QE12, 532 (1976); S. L. McCall and P. M. Platzman, IEEE J. Quantum Electron. QE-21, 1899 (1985).

24. E. Yablonovitch, T. Gmitter, J. P. Harbison, and R. Bhat, Appl. Phys. Lett. 51, 222 (1987)

25. I. Schnitzer, E. Yablonovitch, C. Caneau, and T. J. Gmitter, Appl. Phys. Lett. (to be published). 